\title{
Srategie per la prevenzione del rischio in medicina
}

\section{F. Venneri}

\author{
Clinical Risk Manager, ASL 10 Firenze
}

\section{Introduzione}

L'evento sentinella ovvero quell'evento avverso di particolare gravità e potenzialmente indicativo di un serio malfunzionamento del sistema è accadimento quanto mai probabile e pertanto una risposta correttiva è auspicata e richiesta in maniera impellente. Troppo spesso tale correzione viene cercata nelle responsabilità soggettive, nella colpevolizzazione del singolo e nell'applicazione di provvedimenti puntivi i più diversi. Una risposta comunque così orientata non riuscirà mai a risolvere il problema all'origine, indifferentemente dalla metodologia inquisitoria utilizzata per l'analisi dell'evento. La migliore proposta di analisi dell'errore che ha condotto al verificarsi di un evento sfavorevole deve necessariamente considerare non solo i protagonisti direttamente coinvolti ma anche le circostanze nelle quali tali protagonisti, gli operatori, esercitano la propria professionalità. Oggi non è più sostenibile attendere il verificarsi di un evento sfavorevole per impostare metodi di miglioramento della qualità dei processi assistenziali, di cura e di performance. Viceversa possiamo, anzi dobbiamo identificare i processi e le procedure che sono ad alto rischio di esitare in un evento sentinella ed impostare attraverso una analisi degli stessi, piani di miglioramento e di correzione affinché tali processi risultino a bassissima o pressoché totale assenza di probabilità di generare un evento sfavorevole. Le strutture organizzative in sanità possono adottare specifiche linee di analisi per l'identificazione di cause e concause a particolare affinità con i processi e procedure foriere di un evento sfavorevole. Errori individuali da parte degli operatori medici, infermieri, farmacisti ecc, dovrebbero sollecitare le valutazioni critiche da parte di una organizzazione sanitaria al fine di identificare proprio i punti deboli del processo o del sistema e viceversa, senza necessariamente operare per la ricerca del colpevole e dell'untore.
I principali sistemi di analisi atti a indirizzare verso metodiche correttive sono:

- La formazione continua dello staff.

- La gestione dellinformazione, della comunicazione, dell'accessibilità all'informazione knowledge-based.

- La misura e la valutazione della performance del sistema rispetto sia ai processi che ai risultati.

- Accreditare e promuovere processi per il personale medico e altri operatori professionisti qualificati per il miglioramento continuo della qualità assistenziale e per la sicurezza del paziente e degli stessi operatori. Pertanto, in analogia al processo di cura in medicina, la gestione medica dei processi e delle procedure assistenziali deve seguire il modello di analisi semeiologica del caso: diagnosi, trattamento e follow-up e prevenzione della recidiva. Questo tipo di approccio detto proattivo nella gestione dei processi assistenziali in sanità ha fornito risultati migliori nella prevenzione di eventi avversi rispetto alla semplice individuazione dell'evento e alla ricerca del colpevole.

Il processo di analisi o risk management di un evento avverso si potrebbe pertanto riassumere come segue:

- Segnalazione dell'evento.

- Analisi della sequenza degli eventi e/o individuazione degli errori attivi e latenti (diagnosi).

- Proporre modelli correttivi (trattamento).

- Prevenire che l'evento accada (prevenzione).

- Mitigare gli effetti di un errore (revisione di una procedura o di un percorso).

- Semplificazione (comunicazione).

- Tecnologia (strumenti di lavoro).

- Implementare il trattamento (screening).

- Follow-up (indicatori di qualità, monitoraggio, controllo). Le strategie per la riduzione del rischio in medicina possono quindi essere rappresentate da:

1. Formazione continua dei singoli e di gruppi.

2. Revisione dei processi di cura.

3. Miglioramento della qualità tecnologica. 
4. Mitigare il cambiamento culturale.

5. Individuare i punti deboli dei singoli, del sistema e dell'organizzazione.

\section{Errare è umano}

Esperti di analisi del fattore umano hanno studiato sin dagli anni '40 le cause e gli effetti degli errori. Inizialmente tale analisi è stata applicata per l'elaborazione e la costruzione di aerei militari ad alta complessità strutturale, successivamente lo studio del fattore umano è stato efficientemente applicato a diversi altri settori come l'organizzazione delle centrali nucleari, nell'ambito della NASA e più recentemente in sanità.

Il miglioramento della sicurezza del paziente è sicuramente una delle principali mission della sanità. A tale scopo i sistemi e le procedure dovranno subire una riorganizzazione tale da rendere l'errore meno probabile per non dire assente poiché è pressoché impossibile pensare di eliminare il rischio di errore. Per raggiungere l'obiettivo "miglioramento della sicurezza del paziente", si dovrà prima conoscere e comprendere l'ambiente in cui l'errore esplica la sua azione, informazione questa che può essere ottenuta dalla semplice osservazione e/o anche dall'esperienza.

Due modalità di revisione delle procedure hanno dimostrato di avere efficacia nel diminuire l'incidenza di errori. La prima modalità è conosciuta come eliminazione dell'errore. Con questa modalità la procedura viene modificata in modo da rendere quasi impossibile l'avvento dell'errore. Un tipico esempio è dato dall'apparecchio per l'anestesia che è stato progettato, ad esempio, in modo da evitare scambi negli accessi dei tubi di erogazione dell'ossigeno e dell'ossido d'azoto. Se l'eliminazione dell'errore non è oltremodo possibile o realizzabile, allora si può tentare con la seconda modalità d'approccio nota come riduzione dell'incidenza di errore di cui si riporta un noto esempio occorso nel 1996: in diversi ospedali la maggioranza del personale infermieristico commetteva errori nella somministrazione di soluzioni concentrate e, in particolare, anche con soluzioni concentrate di cloruro di potassio $(\mathrm{KCl})$ la cui pericolosità è proverbiale. In conseguenza di ciò molti ospedali scelsero di eliminare questo farmaco dalle scorte situate nei singoli reparti come suggerito anche dagli esperti della Farmacopea Americana, agendo quindi con l'estromissione di un operatore sanitario, l'infermiere, dall'usuale iter del processo di cura. In questo esempio, la rimozione di un operatore dalla procedura per la preparazione delle soluzioni parenterali contenenti $\mathrm{KCl}$ porta alla riduzione della probabilità di un errore infermieristico ma costringe a delegare a ciò un altro operatore, ad esempio il farmacista: tutto ciò non previene certamente un possibile errore da parte del nuovo operatore coinvolto nella procedura di preparazione di soluzioni così pericolose.

Una terza modalità d'approccio alla gestione del rischio e dell'errore è rappresentata dal ridisegnare l'intero processo assistenziale per eliminare radicalmente le consequenze dell'errore e poiché gli errori potrebbero accadere in maniera occasionale, la procedura può essere ridisegnata per cogliere gli errori prima che essi possano causare l'eventuale dannoso al paziente ovvero mi-

\section{TABELLA I - PRINCIPI PER IL MIGLIORAMENTO DEI SISTEMI DI LAVORO}

1. Semplificare le procedure

2. Ridurre la dipendenza dai processi mnemonici**

3. Standardizzare

4. Migliorare l'accesso all'informazione

5. Utilizzare funzioni restrittive e obbligazioni

6. Ripensare gli errori

7. Adattare e rimodellare l'orario di lavoro

8. Modellare l'ambiente di lavoro

9. Migliorare la comunicazione

10. Diminuire l'affidabilità dipendente dallo stato di concentrazione e vigilanza

11. Promuovere l'addestramento, le simulazioni e la formazione verso le procedure di sicurezza

12. Scegliere lo staff adatto a tale lavoro

** Strumenti utilizzati per ridurre la dipendenza dai meccanismi mnemonici

- Linee-guida della pratica clinica

- Scheda unica di prescrizione e somministrazione del farmaco e delle consegne

- Clinical Pathway 
gliorando le procedure e i processi proprio al fine di $r i$ durre gli effetti degli errori.

$\mathrm{Ne}$ sono una chiara esemplificazione alcuni dei noti principi di miglioramento dei sistemi di lavoro che vengono riportati nella Tabella I.

\section{Conclusioni}

Ormai è a tutti noto sia che l'errore in medicina è una delle principali problematiche della qualità assistenziale e della sicurezza del paziente sia che l'errore stesso non è assolutamente estinguibile. Per tali motivazioni è nata in sanità una istituzione di ricerca e applicazione sulla gestione del rischio clinico, nota come Governo Clinico. Tale istituzione è dovuta alle osservazioni ed esperienze provenienti da altri settori della vita civile e militare come dall'aviazione, dalle organizzazioni di gestione delle centrali nucleari ecc, ove il coinvolgimento di studiosi di ergonomia e dei fattori umani sono stati coinvolti da tempo.

Oggigiorno, inoltre, sappiamo ormai bene che è assai più importante la conoscenza dell'ambiente dove accade l'errore nella propria organizzazione lavorativa che addivenire alla conoscenza del colpevole, magari additandolo in una sorta di pedissequa e ingiusta, oltreché obsoleta, caccia alle streghe. Ancora, sarà di massima utilità il focalizzare gli sforzi sulle modifiche e sulle correzioni delle procedure e dei sistemi piuttosto che sull'osservazione dei singoli operatori.

Solo attraverso un vero e proprio lavoro di gruppo, ben strutturato (Structured Teamwork), si può pervenire a una reale e sensibile riduzione dell'incidenza dell'errore in medicina. Come è avvenuto in settori della me- dicina dove l'utilità di lavorare in team è più apprezzabile e la probabilità di commettere errori è relativamente maggiore in quanto il lavoro dei singoli operatori è processato in momenti di alta tensione e criticità (l'area dell'emergenza-urgenza ad esempio), si dovrebbe tendere alla migliore strutturazione del gruppo, poiché ciò porta almeno a cinque fondamentali obiettivi:

1. Mantenimento alto e qualificato del clima creativo e organizzativo del gruppo.

2. Applicazione di strategie problem-solving.

3. Comunicazione con i membri del gruppo.

4. Applicazione di soluzioni e gestione dei carichi di lavoro e conflitti.

5. Miglioramento dell'abilità e professionalità dei singoli operatori mediante la formazione continua.

In estrema sintesi: migliorare la peformance clinica equivale a mantenere basso il livello di incidenza di errore.

In sanità oggi l'obiettivo qualitativo generale si raggiunge anche evitando che gli errori abbiano ripercussioni, in senso negativo, sulla sicurezza del paziente e degli operatori. Evitare gli eventi avversi è mandatario ma ciò è possibile solo considerando anche il fattore umano e l'ambiente ove viene data assistenza.

L'esperienza personale di chi scrive permette di affermare che l'utilizzo di check-list, sedute di briefing e debriefing, analisi degli eventi avversi mancati, audit clinici, simulazione pianificata e formazione continua sono alcuni fra i principali strumenti attraverso i quali il Governo Clinico riesce a monitorare e a controllare l'incidenza di errore.

\section{francesco.venneri@asf.toscana.it}

\section{Letture consigliate}

- Ministero della Salute, Sicurezza dei pazienti e gestione del rischio clinico: Manuale per la formazione degli operatori sanitari. Disponibile su:

http://www.ministerosalute.it/imgs/C__17_pubblicazioni_ 640_allegato.pdf

- Reason JT. Managing the Risks of Organizational Accidents, 1997.

- Gitlow H, et al. Tools and Methods for the Improvement of Quality, 1989.
- Reason JT. Human Error: Causes and Consequences, 1990.

- Joint Commission on Accreditation of Healthcare Organizations, Sentinel-Events: Evaluating Cause and Planning Improvement, 1998.

- Leape LL. Error in Medicine.

- Vincent C. Clinical Risk Management: Enhancing Patient Safety, 2001. 